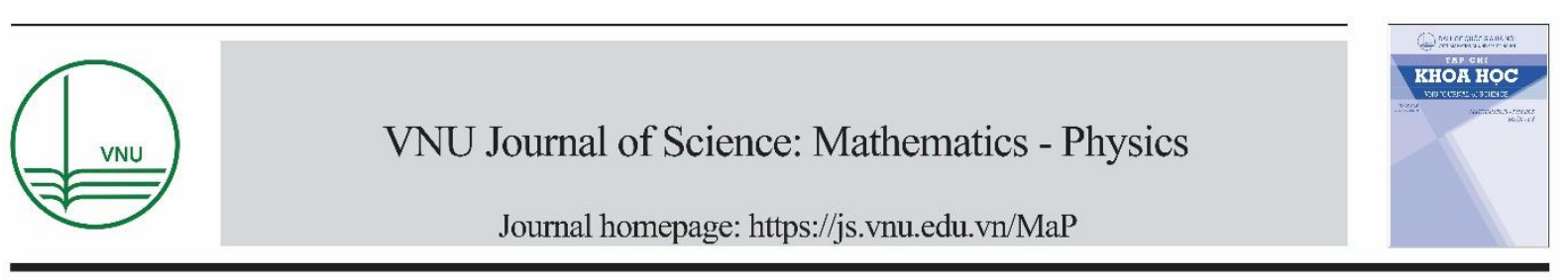

\title{
The Bhabha Scattering in the Randall-sundrum Model
}

\author{
Le Nhu Thuc* \\ Hanoi National University of Education (HNUE), 136 Xuan Thuy, Cau Giay, Hanoi, Vietnam \\ Received 26 September 2018 \\ Revised 26 October 2018; Accepted 17 December 2018
}

\begin{abstract}
The change in other two fermion processes is called Bhabha process. In this paper, we discuss the Bhabha process $e^{+} e^{-} \rightarrow e^{+} e^{-}$in the Randall -Sundrum (RS). We caculated the cross section for photon $\gamma$, boson $\mathrm{Z}$, radion $\phi$ and Higgs $\mathrm{h}$ exchange and evaluated exchange contributions of the Bhabha process in detail.
\end{abstract}

Keywords: DCS, cross-section, Bhabha, radion, Randall-Sundrum.

\section{Introduction}

The RS model [1] can solve the hierarchy problem by localizing all the Standard model (SM) particles on the IR brane. This model predicts two new particles beyond the SM. One is a spin-2 graviton and another is a scalar-field radion which is a metric fluctuation along the extra dimension. The mass of radion is expected to be of the order of $\mathrm{GeV}$. Therefore, the radion is expected to be the first signature of warped extra dimension models in direct search experiments such as the Large Hadron Collider (LHC) [2 - 8].

The Bhabha scattering has been studing in models beyond the SM, and it is also compared ILC250 to LEP2 and LHC [9]. In this paper, we discuss the radion and Higgs exchange contributions in the cross section of the Bhabha scattering in the RS model. We hope that, the Bhabha channel suggests the best way to study radion and Higgs.

\section{The cross-section of the proces $e^{+} e^{-} \rightarrow e^{+} e^{-}$}

The Feynman diagrams of the process $e^{+} e^{-} \rightarrow e^{+} e^{-}$are shown in Fig. 2.1.

\footnotetext{
* Tel.: 84-982004689.

Email: thucln@hnue.edu.vn

https//doi.org/ 10.25073/2588-1124/vnumap.4292
} 


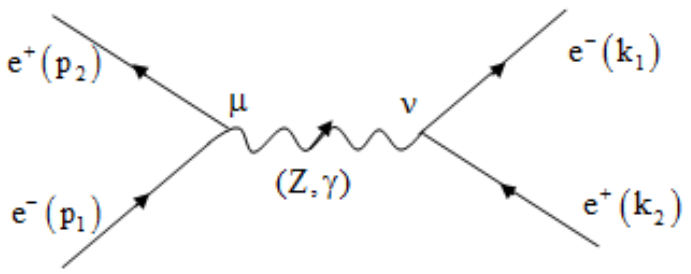

(a)

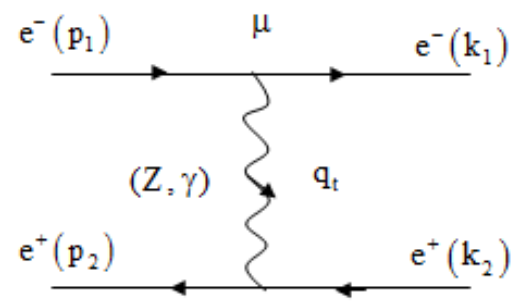

$v$

(c)

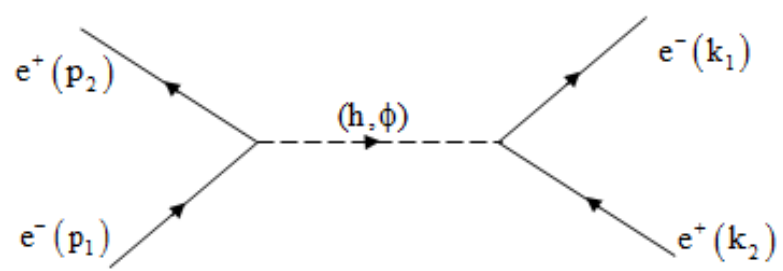

(b)

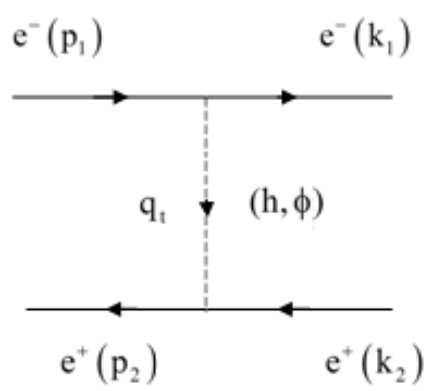

$(d)$

Fig 2.1. The Feynman diagrams for the process $e^{+} e^{-} \rightarrow e^{+} e^{-}$.

Using the Feynman rules for Fig 2.1, the matrix element for the process $e^{+} e^{-} \rightarrow e^{+} e^{-}$is given by:

+ For s-channel (Fig 2.1a, b),

$$
\begin{gathered}
\mathrm{M}_{\mathrm{ty}}=\left[\frac{\mathrm{ie}^{2}}{\mathrm{q}_{\mathrm{s}}^{2}}\right] \mathrm{g}_{\mu v}\left[\overline{\mathrm{u}}\left(\mathrm{k}_{1}, \mathrm{r}_{1}\right) \gamma^{\mu} \mathrm{u}\left(\mathrm{p}_{1}, \mathrm{~s}_{1}\right)\right] \times\left[\overline{\mathrm{v}}\left(\mathrm{p}_{2}, \mathrm{~s}_{2}\right) \gamma^{v} \mathrm{v}\left(\mathrm{k}_{2}, \mathrm{r}_{2}\right)\right] \\
\mathrm{M}_{\mathrm{tz}}=\left[\frac{\mathrm{ig}^{2}}{16 \mathrm{c}_{\mathrm{w}}^{2}\left(\mathrm{q}_{\mathrm{s}}^{2}-\mathrm{m}_{\mathrm{z}}{ }^{2}\right)}\right]\left[\mathrm{g}_{\mu v}-\frac{\mathrm{q}_{\mu} \mathrm{q}_{\mathrm{v}}}{\mathrm{m}_{\mathrm{z}}^{2}}\right]\left[\overline{\mathrm{u}}\left(\mathrm{k}_{1}, \mathrm{r}_{1}\right)\left[\gamma^{\mu}\left(\mathrm{v}_{\mathrm{e}}-\mathrm{a}_{\mathrm{e}} \gamma^{5}\right)\right] \mathrm{u}\left(\mathrm{p}_{1}, \mathrm{~s}_{1}\right)\right] \\
\times\left[\overline{\mathrm{v}}\left(\mathrm{p}_{2}, \mathrm{~s}_{2}\right)\left[\gamma^{v}\left(\mathrm{v}_{\mathrm{e}}-\mathrm{a}_{\mathrm{e}} \gamma^{5}\right)\right] \mathrm{v}\left(\mathrm{k}_{2}, \mathrm{r}_{2}\right)\right] \\
\mathrm{M}_{\mathrm{t \phi}}=\left[\frac{-\mathrm{i}}{\left(\mathrm{q}_{\mathrm{s}}^{2}-\mathrm{m}_{\phi}{ }^{2}\right)}\left(\frac{\mathrm{g}}{2} \frac{\mathrm{m}_{\mathrm{e}}}{\mathrm{m}_{\mathrm{w}}}\right)^{2}(\mathrm{c}+\gamma \mathrm{a})^{2}\right] \overline{\mathrm{u}}\left(\mathrm{k}_{1}, \mathrm{r}_{1}\right) \mathrm{u}\left(\mathrm{p}_{1}, \mathrm{~s}_{1}\right) \overline{\mathrm{v}}\left(\mathrm{p}_{2}, \mathrm{~s}_{2}\right) \mathrm{v}\left(\mathrm{k}_{2}, \mathrm{r}_{2}\right) \\
\mathrm{M}_{\mathrm{th}}=\left[\frac{-\mathrm{i}}{\left(\mathrm{q}_{\mathrm{s}}^{2}-\mathrm{m}_{\mathrm{h}}{ }^{2}\right)}\left(\frac{\mathrm{g}}{2} \frac{\mathrm{m}_{\mathrm{e}}}{\mathrm{m}_{\mathrm{w}}}\right)^{2}(\mathrm{~d}+\gamma \mathrm{b})^{2}\right] \overline{\mathrm{u}}\left(\mathrm{k}_{1}, \mathrm{r}_{1}\right) \mathrm{u}\left(\mathrm{p}_{1}, \mathrm{~s}_{1}\right) \overline{\mathrm{v}}\left(\mathrm{p}_{2}, \mathrm{~s}_{2}\right) \mathrm{v}\left(\mathrm{k}_{2}, \mathrm{r}_{2}\right)
\end{gathered}
$$

+ For t-channel (Fig 2.1c, d),

$$
\mathrm{M}_{\mathrm{t} \gamma}^{+}=\left[\frac{-\mathrm{i} \mathrm{e}^{2}}{\mathrm{q}_{\mathrm{t}}^{2}}\right] \mathrm{g}_{\mu^{\prime} \mathrm{v}^{\prime}}\left[\overline{\mathrm{v}}\left(\mathrm{k}_{2}, \mathrm{r}_{2}\right) \gamma^{\mu^{\prime}} \mathrm{v}\left(\mathrm{p}_{2}, \mathrm{~s}_{2}\right)\right] \times\left[\overline{\mathrm{u}}\left(\mathrm{p}_{1}, \mathrm{~s}_{1}\right) \gamma^{v^{\prime}} \mathrm{u}\left(\mathrm{k}_{1}, \mathrm{r}_{1}\right)\right]
$$




$$
\begin{aligned}
& \mathrm{M}_{\mathrm{tz}}^{+}=\left[\frac{\mathrm{ig}^{2}}{16 \mathrm{c}^{2}{ }_{\mathrm{w}}\left(\mathrm{q}_{\mathrm{t}}{ }^{2}-\mathrm{m}_{\mathrm{z}}{ }^{2}\right)}\right] \times\left[\mathrm{g}_{\mu^{\prime} \mathrm{v}^{\prime}}-\frac{\mathrm{q}_{\mu^{\prime}} \mathrm{q}_{\mathrm{v}^{\prime}}}{\mathrm{m}_{\mathrm{Z}}^{2}}\right] \\
& \times\left[\overline{\mathrm{v}}\left(\mathrm{k}_{2}, \mathrm{r}_{2}\right)\left[\gamma^{\mathrm{v}^{\prime}}\left(\mathrm{v}_{\mathrm{e}}+\mathrm{a}_{\mathrm{e}} \gamma^{5}\right)\right] \mathrm{v}\left(\mathrm{p}_{2}, \mathrm{~s}_{2}\right)\right]\left[\overline{\mathrm{u}}\left(\mathrm{p}_{1}, \mathrm{~s}_{1}\right)\left[\gamma^{\mu^{\prime}}\left(\mathrm{v}_{\mathrm{e}}+\mathrm{a}_{\mathrm{e}} \gamma^{5}\right)\right] \mathrm{u}\left(\mathrm{k}_{1}, \mathrm{r}_{1}\right)\right] \\
& \mathrm{M}_{\mathrm{t} \phi}^{+}=\left[\frac{\mathrm{i}}{\left(\mathrm{q}_{\mathrm{t}}{ }^{2}-\mathrm{m}_{\phi}{ }^{2}\right)}\left(\frac{\mathrm{g}}{2} \frac{\mathrm{m}_{\mathrm{e}}}{\mathrm{m}_{\mathrm{w}}}\right)^{2}(\mathrm{c}+\gamma \mathrm{a})^{2}\right] \overline{\mathrm{v}}\left(\mathrm{k}_{2}\right) \mathrm{v}\left(\mathrm{p}_{2}\right) \overline{\mathrm{u}}\left(\mathrm{p}_{1}\right) \mathrm{u}\left(\mathrm{k}_{1}\right) \\
& \mathrm{M}_{\mathrm{th}}^{+}=\left[\frac{\mathrm{i}}{\left(\mathrm{q}_{\mathrm{t}}{ }^{2}-\mathrm{m}_{\mathrm{h}}{ }^{2}\right)}\left(\frac{\mathrm{g}}{2} \frac{\mathrm{m}_{\mathrm{e}}}{\mathrm{m}_{\mathrm{w}}}\right)^{2}(\mathrm{~d}+\gamma \mathrm{b})^{2}\right] \overline{\mathrm{v}}\left(\mathrm{k}_{2}\right) \mathrm{v}\left(\mathrm{p}_{2}\right) \overline{\mathrm{u}}\left(\mathrm{p}_{1}\right) \mathrm{u}\left(\mathrm{k}_{1}\right)
\end{aligned}
$$

From (1-8), we have

$$
\begin{aligned}
& \left|\mathbf{M}_{\mathrm{s} \gamma}\right|^{2}=\frac{16 e^{4}}{q_{s}^{4}}\left[2\left(p_{1} k_{2}\right)\left(p_{2} k_{1}\right)+2\left(p_{1} k_{1}\right)\left(p_{2} k_{2}\right)-\left(p_{1} p_{2}\right)\left(k_{1} k_{2}\right)\right. \\
& \left.+2\left(\left(p_{2} p_{1}\right)+\left(k_{1} k_{2}\right)\right)+m_{e}^{2}\left(\left(p_{2} k_{2}\right)+\left(p_{1} k_{1}\right)\right)+m_{e}^{4}\right] \\
& \left|\mathrm{M}_{\mathrm{sz}}\right|^{2}=16\left[\frac{-g^{4}}{256 \mathrm{c}^{4}{ }_{w}\left(\mathrm{q}_{s}{ }^{2}-\mathrm{m}_{z}{ }^{2}\right)^{2}}\right]\left\{2\left(p_{2} k_{1}\right)\left(p_{1} k_{2}\right)+2\left(k_{1} k_{2}\right)\left(p_{2} p_{1}\right)-3\left(p_{1} k_{1}\right)\left(p_{2} k_{2}\right)\right. \\
& -m e^{2}\left(p_{1} k_{1}\right)++m_{e}^{4}-\frac{2}{m_{z}^{2}}\left[2\left(p_{1} k_{2}\right)\left(q_{s} k_{1}\right)\left(q_{s} p_{2}\right)+\left(k_{1} k_{2}\right)\left(q_{s} p_{1}\right)\left(q_{s} p_{2}\right)\right. \\
& +\left(p_{1} p_{2}\right)\left(q_{s} k_{1}\right)\left(q_{s} k_{2}\right)-2\left(p_{2} k_{2}\right)\left(q_{s} k_{1}\right)\left(q_{s} k_{2}\right)-2 m e^{2}\left(q_{s} k_{1}\right)\left(q_{s} p_{1}\right) \\
& \left.\left.-\left(p_{1} k_{1}\right)\left(p_{2} k_{2}\right)+m e^{2}\left(p_{1} k_{1}\right)+m e^{4}\right)\right]+\frac{1}{m_{z}^{4}}\left[4\left(q_{s} p_{1}\right)\left(q_{s} p_{2}\right)\left(q_{s} k_{1}\right)\left(q_{s} k_{2}\right)\right. \\
& -2\left(q_{s}^{2}\right)\left(q_{s} p_{1}\right)\left(q_{s} k_{1}\right)\left(p_{2} k_{2}\right)-2 m_{e}^{2}\left(q_{s}^{2}\right)\left(q_{s} p_{1}\right)\left(q_{s} k_{1}\right)-2\left(p_{1} k_{1}\right)\left(q_{s} p_{2}\right)\left(q_{s} k_{2}\right) \\
& \left.\left.+\left(p_{1} k_{1}\right)\left(p_{2} k_{2}\right)\left(q_{s}^{2} q_{s}^{2}\right)+m_{e}^{2}\left(p_{1} k_{1}\right)\left(q_{s}^{2} q_{s}^{2}\right)+m_{e}^{4}\left(q_{s}^{2} q_{s}^{2}\right)\right]\right\} \\
& \left|\mathbf{M}_{\mathrm{s} \phi}\right|^{2}=16\left[\frac{-1}{\left(\mathrm{q}_{s}{ }^{2}-\mathrm{m}_{\phi}{ }^{2}\right)^{2}}\left(\frac{\mathrm{g}}{2} \frac{m_{e}}{m_{w}}\right)^{4}(\mathrm{c}+\gamma \mathrm{a})^{4}\right]\left\{\mathrm{p}_{2} \mathrm{p}_{1}-m_{e}{ }^{2}\right\}\left\{k_{1} k_{2}-m_{e}{ }^{2}\right\}, \\
& \left|\mathrm{M}_{\mathrm{sh}}\right|^{2}=16\left[\frac{-1}{\left(\mathrm{q}_{s}{ }^{2}-\mathrm{m}_{h}{ }^{2}\right)^{2}}\left(\frac{\mathrm{g}}{2} \frac{m_{e}}{m_{w}}\right)^{4}(\mathrm{~d}+\gamma \mathrm{b})^{4}\right]\left\{\mathrm{p}_{2} \mathrm{p}_{1}-m_{e}{ }^{2}\right\}\left\{k_{1} k_{2}-m_{e}{ }^{2}\right\} \\
& \left|\mathbf{M}_{t \gamma}\right|^{2}=\frac{16 e^{4}}{q_{s}^{4}}\left[2\left(p_{1} k_{2}\right)\left(p_{2} k_{1}\right)-3\left(p_{1} k_{1}\right)\left(p_{2} k_{2}\right)+2\left(p_{1} p_{2}\right)\left(k_{1} k_{2}\right)\right. \\
& \left.+\left(p_{2} k_{2}\right)\left(p_{1} k_{1}\right)+m_{e}^{2}\left(\left(p_{2} p_{1}\right)+\left(k_{1} k_{2}\right)\right)+m_{e}^{4}\right]
\end{aligned}
$$




$$
\begin{aligned}
& \left|\mathbf{M}_{\mathrm{tz}}\right|^{2}=16\left[\frac{-g^{4}}{256 \mathrm{c}^{4}{ }_{w}\left(\mathrm{q}_{s}{ }^{2}-\mathrm{m}_{z}{ }^{2}\right)^{2}}\right]\left\{2\left(p_{2} k_{1}\right)\left(p_{1} k_{2}\right)-3\left(k_{1} k_{2}\right)\left(p_{2} p_{1}\right)\right. \\
& +2\left(p_{1} k_{1}\right)\left(p_{2} k_{2}\right)-m e^{2}\left(p_{2} k_{2}\right)+m_{e}{ }^{4}-\frac{2}{m_{z}^{2}}\left[2\left(p_{1} k_{2}\right)\left(q_{t} k_{1}\right)\left(q_{t} p_{2}\right)\right. \\
& -2\left(k_{1} k_{2}\right)\left(q_{t} p_{1}\right)\left(q_{t} p_{2}\right)+\left(p_{1} p_{2}\right)\left(q_{t} k_{1}\right)\left(q_{t} k_{2}\right)-\left(p_{2} k_{2}\right)\left(q_{t} k_{1}\right)\left(q_{t} k_{2}\right) \\
& +\left(p_{1} k_{1}\right)\left(q_{t} p_{2}\right)\left(q_{t} k_{2}\right)-2 m_{e}^{2}\left(q_{t} p_{2}\right)\left(q_{t} p_{1}\right)-\left(p_{1} p_{2}\right)\left(k_{1} k_{2}\right)+m_{e}^{2}\left(p_{1} p_{2}\right) \\
& \left.\left.+m e^{4}\right)\right]+\frac{1}{m_{z}{ }^{4}}\left[4\left(q_{t} p_{1}\right)\left(q_{t} p_{2}\right)\left(q_{t} k_{1}\right)\left(q_{t} k_{2}\right)-2\left(q_{t}^{2}\right)\left(q_{t} p_{1}\right)\left(q_{t} p_{2}\right)\left(k_{1} k_{2}\right)\right. \\
& -2 m_{e}^{2}\left(q_{t}^{2}\right)\left(q_{t} p_{1}\right)\left(q_{t} p_{2}\right)-2\left(p_{1} p_{2}\right)\left(q_{t} k_{1}\right)\left(q_{t} k_{2}\right)+\left(p_{1} p_{2}\right)\left(k_{1} k_{2}\right)\left(q_{t}^{2} q_{t}^{2}\right) \\
& \left.\left.+m_{e}^{2}\left(p_{1} p_{2}\right)\left(q_{t}^{2} q_{t}^{2}\right)+m_{e}^{4}\left(q_{t}^{2} q_{t}^{2}\right)\right]\right\} \\
& \left|M_{t \phi}\right|^{2}=16\left[\frac{-1}{\left(\mathrm{q}_{\mathrm{s}}{ }^{2}-\mathrm{m}_{\phi}{ }^{2}\right)^{2}}\left(\frac{\mathrm{g}}{2} \frac{\mathrm{m}_{\mathrm{e}}}{\mathrm{m}_{\mathrm{w}}}\right)^{4}(\mathrm{c}+\gamma \mathrm{a})^{4}\right]\left\{\mathrm{k}_{1} \mathrm{p}_{1}+\mathrm{m}_{\mathrm{e}}{ }^{2}\right\}\left\{\mathrm{k}_{2} \mathrm{p}_{2}+\mathrm{m}_{\mathrm{e}}{ }^{2}\right\} \\
& \left|\mathrm{M}_{\mathrm{th}}\right|^{2}=16\left[\frac{-1}{\left(\mathrm{q}_{t}{ }^{2}-\mathrm{m}_{h}{ }^{2}\right)^{2}}\left(\frac{\mathrm{g}}{2} \frac{m_{e}}{m_{w}}\right)^{4}(\mathrm{~d}+\gamma \mathrm{b})^{4}\right]\left\{k_{1} \mathrm{p}_{1}+m_{e}{ }^{2}\right\}\left\{k_{2} \mathrm{p}_{2}+m_{e}{ }^{2}\right\}
\end{aligned}
$$

The cross section for process $e^{+} e^{-} \rightarrow e^{+} e^{-}$is given by

$$
\frac{d \sigma}{d \Omega}=\frac{1}{64 \pi^{2} s} \frac{|\vec{k}|}{|\vec{p}|}\left|M_{f i}\right|^{2} S \text {. }
$$

where $\sqrt{s}$ is the center-of-mass energy, $\left|M_{f i}\right|^{2}$ is the square of matrix element, $d \Omega=d(\cos \theta) d \varphi ; \theta \in[0, \pi] ; \varphi \in[0,2 \pi]$.

We choose $\mathrm{m}_{\mathrm{e}}=0,00051 \mathrm{GeV}, \mathrm{m}_{\mathrm{w}}=80 \mathrm{GeV}, \mathrm{m}_{\mathrm{z}}=91,2 \mathrm{GeV}, \mathrm{m}_{\phi}=10 \mathrm{GeV}, \mathrm{v}_{0}=246 \mathrm{GeV}$, $\lambda_{\phi}=5000, \quad \gamma=\frac{\mathrm{v}_{0}}{\lambda_{\phi}}=\frac{123}{2500}, \mathrm{~S}_{\mathrm{w}}=0,231, \mathrm{C}_{\mathrm{w}}=\sqrt{1-\mathrm{S}_{\mathrm{w}}^{2}}, \quad \mathrm{~g}=\frac{2 \mathrm{~m}_{\mathrm{w}}}{\mathrm{v}_{0}} \mathrm{C}_{\mathrm{w}}, \mathrm{a}_{\mathrm{e}}=\frac{-1}{2}, \mathrm{v}_{\mathrm{e}}=-\frac{1}{2}+2 \mathrm{~s}_{\mathrm{w}}^{2}$, $\mathrm{a}=\frac{\cos \theta}{\mathrm{Z}}, \mathrm{c}=\sin \theta-\frac{6 \xi \gamma}{\mathrm{Z}} \cos \theta, \xi=\frac{1}{6}, \mathrm{Z}=\sqrt{\left(1+6 \xi \gamma^{2}(1-6 \xi)\right)}$. The $\cos \theta$ of the $e^{+} e^{-} \rightarrow e^{+} e^{-}$ differential cross section is shown in figure 2.2 and figure 2.3 at $\sqrt{s}=3000 \mathrm{GeV}$.

Figure 2.a, $\mathrm{b}$ show the DCS via radion and Higgs exchange for s-channel, does not depend on $\cos \theta$ . The DCS is $4,2.10^{-29}$ pbarn a for radion exchange contribution and $0,7.10^{-23}$ pbarn for Higgs exchange contribution. The DCS via radion and Higgs exchange for $\mathrm{t}$ channel depend on $\cos \theta$. However, the DSC decreases very small while $\cos \theta$ increases from -1 to 1 (Fig $2.3 \mathrm{c}, \mathrm{d}$ ).

For photon and $\mathrm{Z}$ boson exchange contributions, the DCS is very large, and it is much larger than for radion and Higgs exchange contributions. 


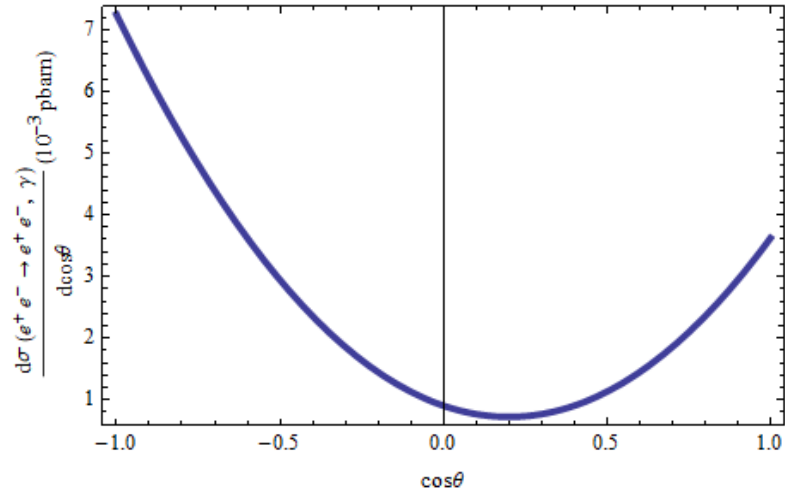

a)

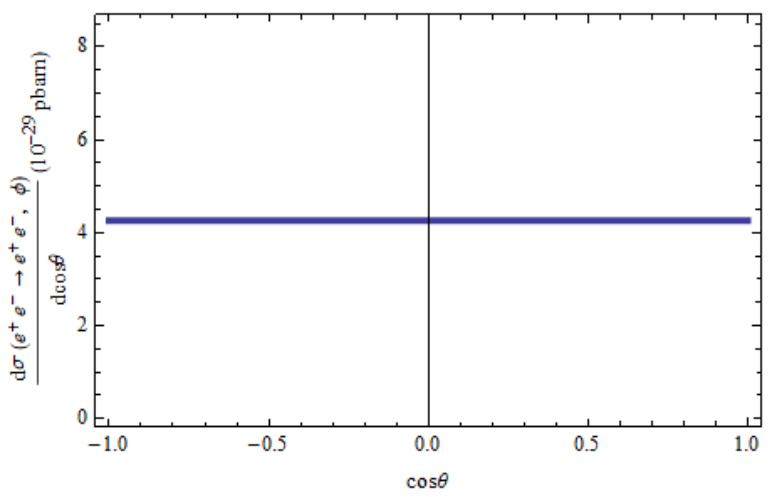

c)

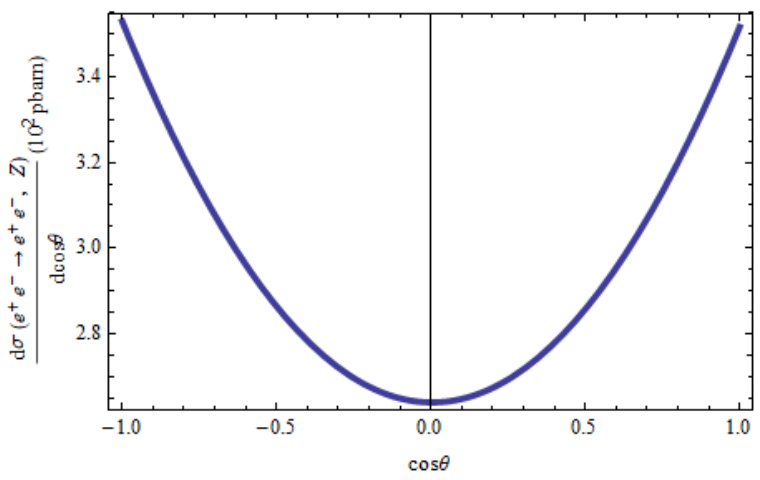

b)

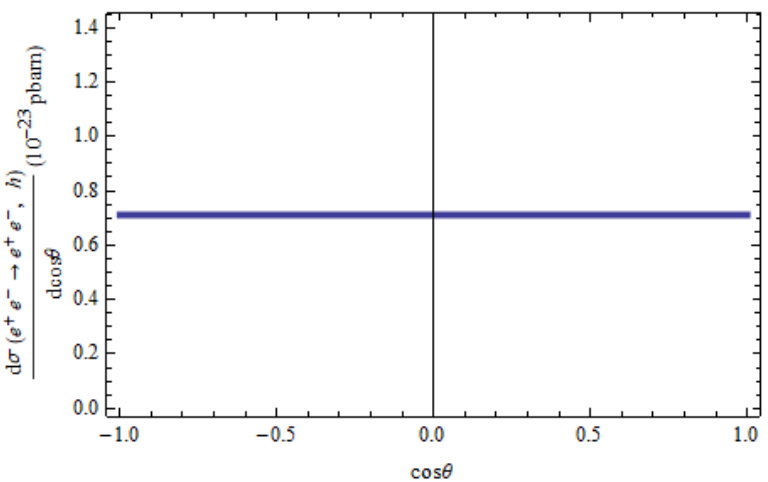

d)

Fig2.2. The $e^{+} e^{-} \rightarrow e^{+} e^{-}$differential cross section via photon (a), $\mathrm{Z}$ boson (b), radion (c), Higgs (d) exchange for s-channel as a function of $\cos \theta$.

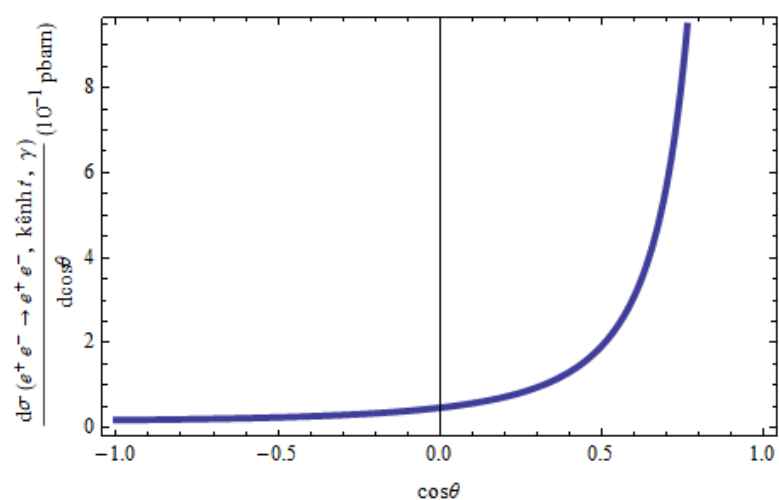

a)

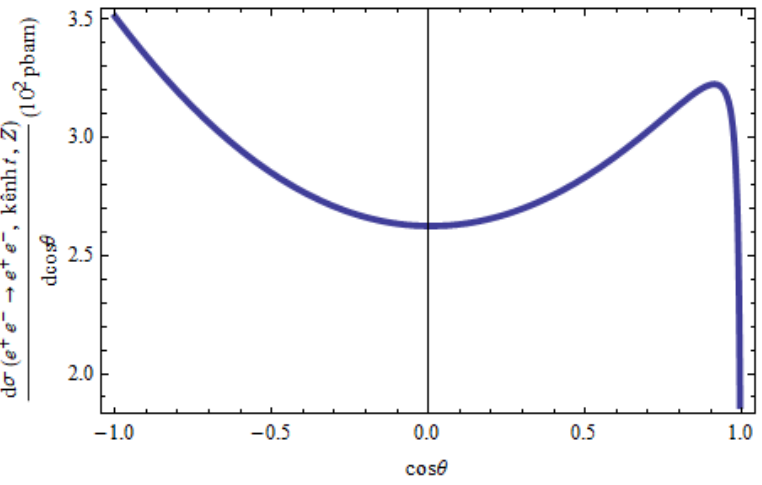

b) 


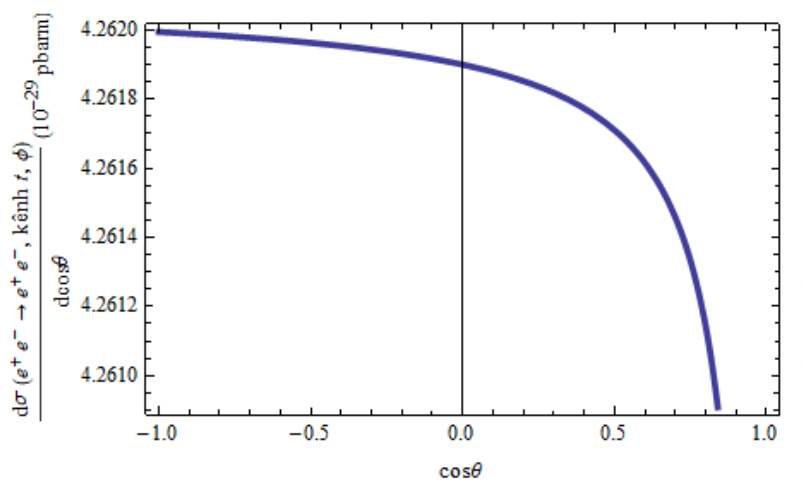

c)

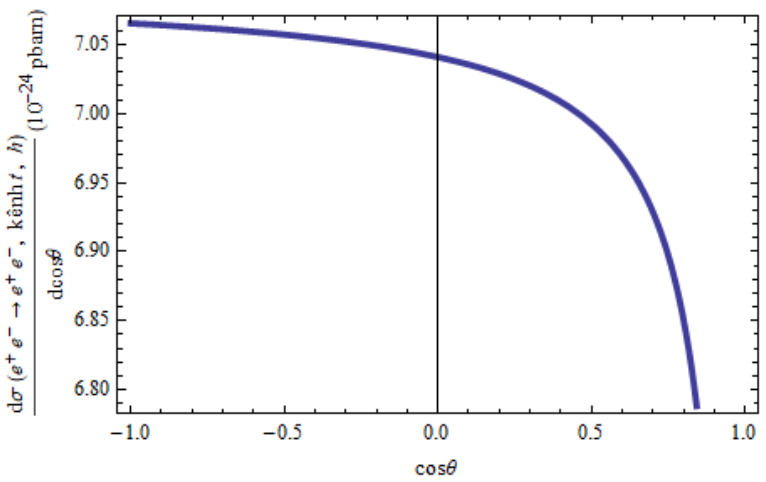

d)

Fig 2.3. The $e^{+} e^{-} \rightarrow e^{+} e^{-}$differential cross - section via photon (a), $\mathrm{Z}$ boson (b), radion (c), Higgs (d) exchange for t-channel as a function of $\cos \theta$.

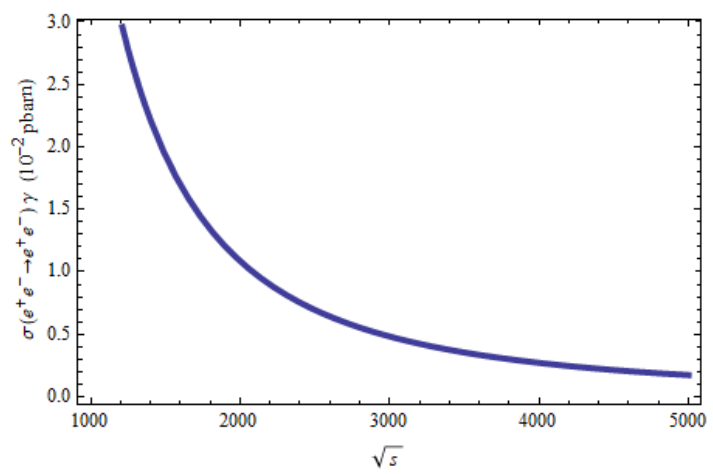

a)

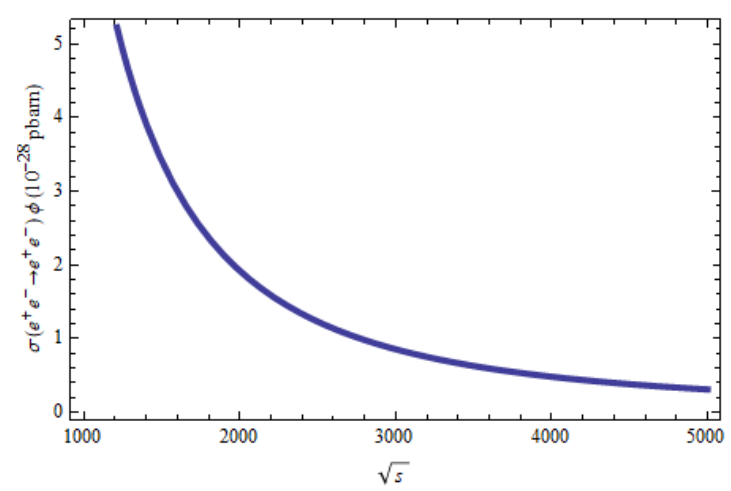

c)

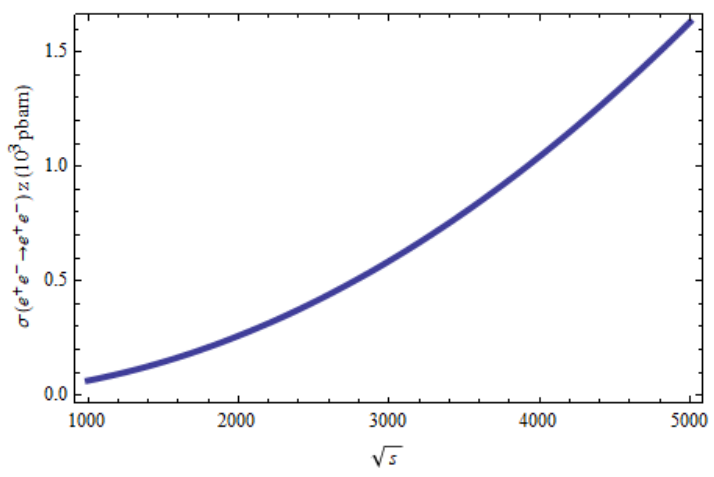

b)

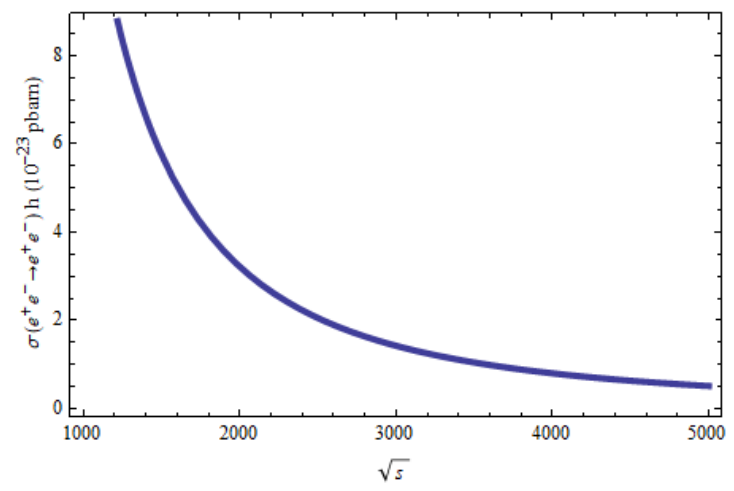

d)

Fig 2.4. The $\mathrm{e}^{+} \mathrm{e}^{-} \rightarrow \mathrm{e}^{+} \mathrm{e}^{-}$total cross section via photon (a), $\mathrm{Z}$ boson (b), radion (c), Higgs (d) exchange for schannel as a function of $\sqrt{\mathrm{s}}$. 


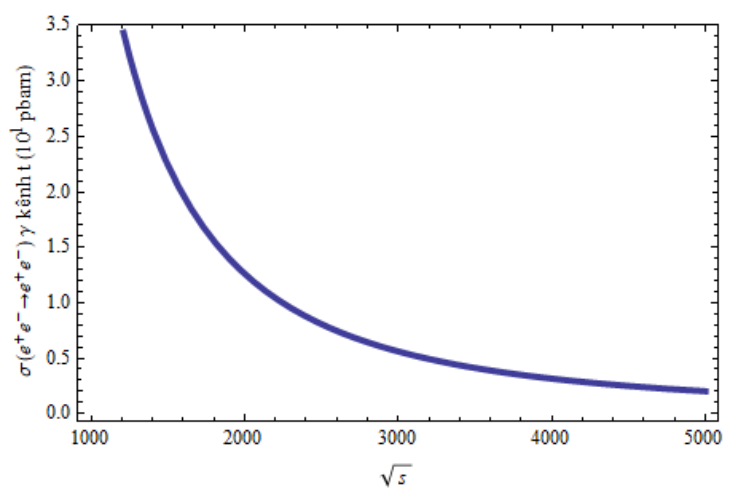

a)

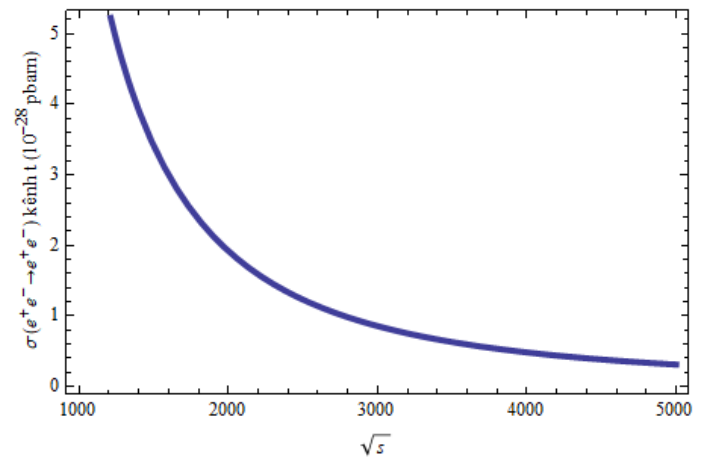

c)

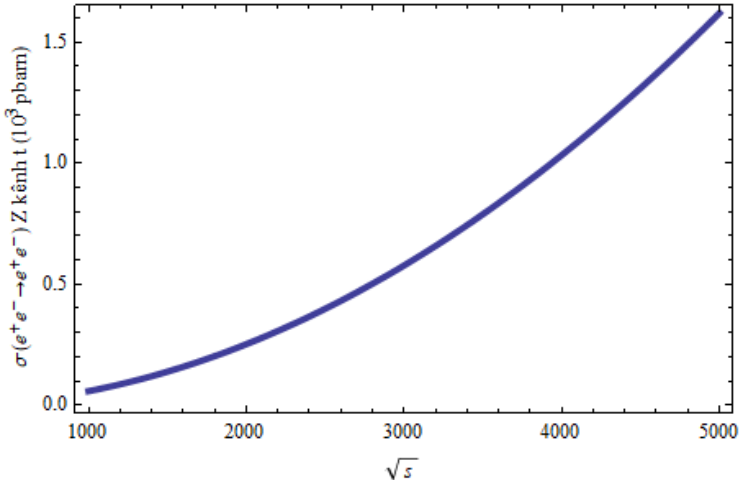

b)

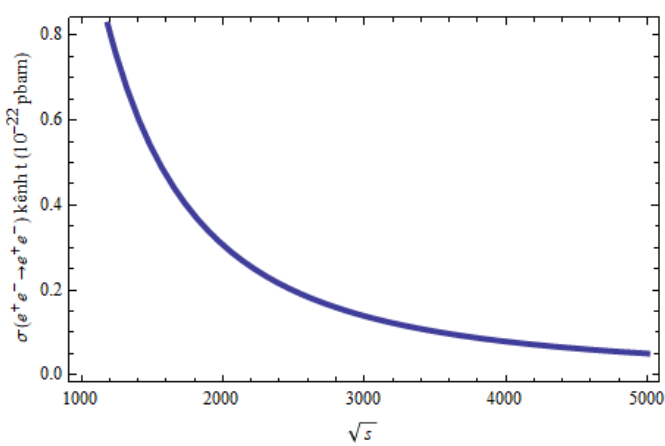

d)

Fig 2.5. The $\mathrm{e}^{+} \mathrm{e}^{-} \rightarrow \mathrm{e}^{+} \mathrm{e}^{-}$total cross section via photon (a), Z boson (b),radion (c), Higgs (d) exchange for schannel as a function of $\sqrt{\mathrm{s}}$.

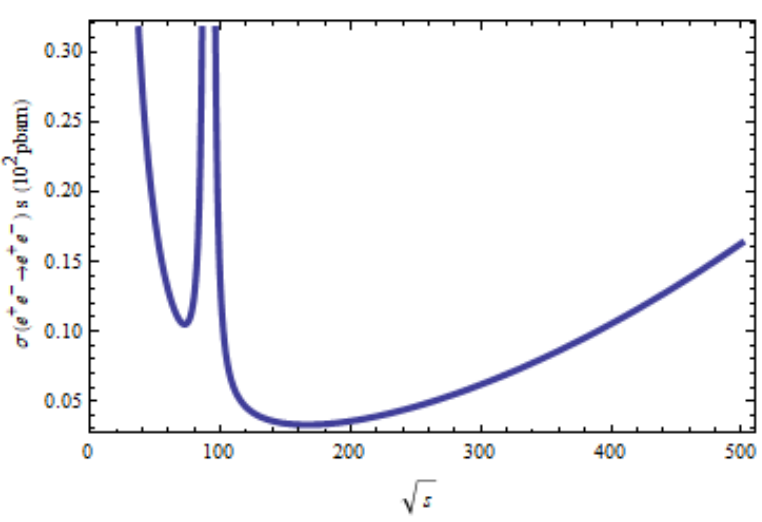

a)

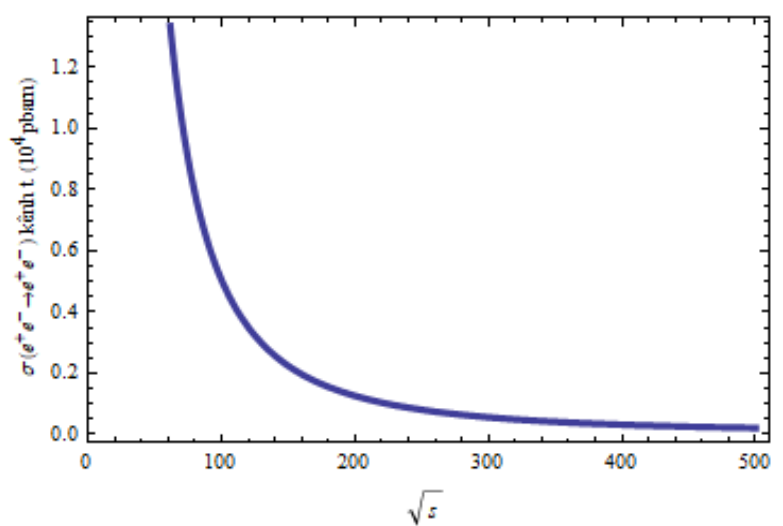

b)

Fig 2.6. The $\mathrm{e}^{+} \mathrm{e}^{-} \rightarrow \mathrm{e}^{+} \mathrm{e}^{-}$total cross section as a function of $\sqrt{s}$ for $\mathrm{s}(\mathrm{a}), \mathrm{t}(\mathrm{b})$-channel. 
Next, we plotted the $\sqrt{s}$ dependence of the $e^{+} e^{-} \rightarrow e^{+} e^{-}$cross section. The results are shown in figure 2.4-2.6. Here, we see that, the total cross section for photon, radion and Higgs exchange contributions decrease while $\sqrt{\mathrm{s}}$ increases from $1000 \mathrm{GeV}$ to $5000 \mathrm{GeV}$. For $\mathrm{Z}$ boson exchange contribution, the total cross section increases while $\sqrt{s}$ increases from $1000 \mathrm{GeV}$ to $5000 \mathrm{GeV}$. However, in low energy region $(\sqrt{s}<100 G e V)$, the $e^{+} e^{-} \rightarrow e^{+} e^{-}$total cross section for s (a), $\mathrm{t}$ (b) channel is very large.

\section{Conclusion}

In this paper, we evaluated the photon, $\mathrm{Z}$ boson, radion and Higgs exchange contributions in the Randall-Sundrum model in detail. The numerical results show that, the radion and Higgs exchange contributions are very small, it is much smaller than the contribution of the photon and $\mathrm{Z}$ cases. This suggests that if radion and Higgs were produced in the high energy region, so the ability to observe them is possible by Bhabha scattering.

\section{References}

[1] L. Randall and R. Sundrum, Phys. Rev. Lett. 83, 3370, 1999.

[2] H. Davoudiasl, T. McElmurry and A. Soni, Phys. Rev. D82, 115028, 2010.

[3] V. P. Goncalves and W. K. Sauter, Phys. Rev. D82, 056009, 2010.

[4] W. -J. Zhang, W. -G. Ma, R. -Y. Zhang, X. -Z. Li, L. Guo, and C. Chen, Phys. Rev. D92, 116005, 2015, [arXiv:1512.01766].

[5] C. Cai, Z.-H. Yu and H.-H. Zhang, Phys. Rev. D93. 075033, 2016.

[6] F. Abu-Ajamieh, R. Houtz, and R. Zheng, [arXiv: 1607.01464v1 [hep-ph]], 2016.

[7] The CMS Collaboration, CERN, August 2016.

[8] The CMS Collaboration, CERN, September 2016.

[9] F. Richard, [ arXiv:1804.02846 [hep-ex]], 2018. 